\title{
Relative dispersion in fully developed turbulence: The Richardson's Law and Intermittency Corrections
}

\author{
G. Boffetta ${ }^{1}$ and I. M. Sokolov ${ }^{2}$ \\ ${ }^{1}$ Dipartimento di Fisica Generale and INFM, \\ Università di Torino, \\ Via Pietro Giuria 1, I-10125 Torino, Italy \\ 2 Theoretische Polymerphysik, Universität Freiburg, \\ Hermann-Herder Straße 3 D-79104 Freiburg i.Br., Germany
}

\begin{abstract}
Relative dispersion in fully developed turbulence is investigated by means of direct numerical simulations. Lagrangian statistics is found to be compatible with Richardson description although small systematic deviations are found. The value of the Richardson constant is estimated as $C_{2} \simeq$ 0.55, in a close agreement with recent experimental findings [S. Ott and J. Mann J. Fluid Mech. 422, 207 (2000)]. By means of exit-time statistics it is shown that the deviations from Richardson's law are a consequence of Eulerian intermittency. The measured Lagrangian scaling exponents require a set of Eulerian structure function exponents $\zeta_{p}$ which are remarkably close to standard ones known for fully developed turbulence.
\end{abstract}

The statistics of two particle dispersion is historically one of the first issues which has been quantitatively addressed in the study of fully developed turbulence. This was done by Richardson, in a pioneering work on the properties of dispersion in the atmosphere in 1926 [1] , 15 years before the theoretical development by Kolmogorov and Obukhov [2]. Despite this fact, there are still relatively few experimental studies on turbulent Lagrangian dispersion. This is essentially due to the difficulties to obtain Lagrangian trajectories in fully developed turbulent flow. The first studies where done in geophysical flows (see [2] for a review) in which Lagrangian tracers are more easily followed. Recently, the problem was approached in laboratory experiments [3. 3 . but the results are still not conclusive. Moreover, most of the numerical studies of relative dispersion rely on kinematic simulations in synthetic flows [5,6]. Direct numerical simulation have been done mostly for two-dimensional turbulence [7, 8].

The scope of this Letter is to contribute to the understanding of relative dispersion by means of direct numerical simulations of three dimensional turbulence. In what follows we show the qualitative validity of the Richardson's description, and discuss its limitations as posed by Lagrangian intermittency, whose properties will be investigated in detail.

Richardson's original description of relative dispersion is based on a diffusion equation for the probability density function of pair separation $p(\mathbf{r}, t)$ which in the isotropic case can be written as

$$
\frac{\partial p(\mathbf{r}, t)}{\partial t}=\frac{1}{r^{2}} \frac{\partial}{\partial r} r^{2} K(r) \frac{\partial p(\mathbf{r}, t)}{\partial r} .
$$

The turbulent eddy diffusivity was empirically established by Richardson to follow the "four-thirds law" $K(r) \propto r^{4 / 3}$. This law is a direct consequence of the small-scale velocity statistics, as was first recognized by Obukhov [9]. Thus, for $r$ within the inertial range, the dimensional analysis gives

$$
K(r)=k_{0} \varepsilon^{1 / 3} r^{4 / 3}
$$

where $\varepsilon$ is the mean energy dissipation and $k_{0}$ a dimensionless constant. We remark that (2) does not imply that a finite energy flux is necessary for particle dispersion. Indeed, particle separation is observed also in pseudo-turbulent synthetic Gaussian velocity field [5,6[10]. Using (2), the solution of (11) for $\delta$-distribution initial condition has the well known form

$$
p(\mathbf{r}, t)=\frac{A}{\left(k_{0} t\right)^{3} \varepsilon} \exp \left(-\frac{9 r^{2 / 3}}{4 k_{0} \varepsilon^{1 / 3} t}\right)
$$

where $A=2187 / 2240 \pi^{3 / 2}$ is a normalizing factor. The most important feature of the Richardson distribution (3) is non-Gaussianity with a very pronounced peak at the origin and rather fat tails. In the past, alternative distributions have been proposed [11,12. In particular Batchelor [11] suggested a Gaussian distribution as a consequence of a diffusivity which depends only on averaged quantities. Because the available data is scarce, there is still no general consensus on the real form of pair separation pdf. Recent experimental works [3, 1 are in favor of (3).

The possibility to describe the dispersion process by means of a diffusion equation is based on two physical assumptions. The first is that the velocity field is short correlated in time. Indeed, in the limit of velocity field $\delta$-correlated in time (the so-called Kraichnan model of turbulence) the diffusion equation of the type of Eq.(1) becomes exact 13,14. The effects of finite correlation time have been recently discussed in [8, 15, 16].

The second assumption, which is one of the points discussed in this Letter, is that the dispersion process is self-similar in time, i.e. the scaling exponents of the moments of the separation

$$
R^{2 n}(t) \equiv\left\langle r^{2 n}(t)\right\rangle=C_{2 n} \varepsilon^{n} t^{\alpha_{2 n}}
$$


have the values $\alpha_{2 n}=3 n / 2$, as following from dimensional analysis. If this is the case, a single number, such as the Richardson constant $C_{2}$ is sufficient to parameterize turbulent dispersion. There is still a large uncertainty on the value of $C_{2}$, ranging from $O\left(10^{-2}\right)-O\left(10^{-1}\right)$ for kinematic simulations 5.6 to $O(1)$ or more in the case of closure predictions [2]. A recent experimental investigation gives the value $C_{2}=0.5$ [ 4 . The hypothesis of self-similarity is reasonable with a self-affine Eulerian velocity, such as in the case of two-dimensional inverse cascade turbulence [8]. A recent analysis of a kinematic model with synthetic velocity field has shown that Lagrangian self-similarity can be broken in presence of Eulerian intermittency. In this case the exponents $\alpha_{n}$ have been found in agreement with the prediction of a multifractal approach for Lagrangian statistics. In particular, the second moment of relative dispersion is not affected by intermittency, i.e. $\alpha_{2}=3$ [10], essentially because it is proportional to $\varepsilon^{1}$. We remind that Lagrangian intermittency has been observed also in the case of the socalled strong anomalous diffusion [17]. Although in that case the mechanism leading to intermittency is different (there is no scaling invariant flow), the implication for Lagrangian description is identical, i.e. the process can not be described by a Fokker-Planck equation like (伍).

We now turn to our numerical procedure. The turbulent velocity field is generated by direct integration of Navier-Stokes equation in a periodic box of size $L=$ $2 \pi$. The integration is done on a parallel computer by means of a pseudo-spectral code at resolution $256^{3}$ with $R e_{\lambda} \simeq 200$. Energy is injected into the flow by keeping the total energy in each of the two first wavenumber shells constant [18] and is removed by a second-order hyperviscous dissipation. Time integration is performed with a second-order Runge-Kutta scheme. In Figure 1 we plot the energy spectrum which shows a well developed Kolmogorov power-law scaling. A small "bump" at $k \gtrsim 20$ is the signature of a bottleneck effect [19] as a consequence of hyperviscosity. In the inset of Figure 1 we show the third order longitudinal structure function $S_{3}(x)=\left\langle\delta u(x)^{3}\right\rangle$ compensated with the theoretical prediction $S_{3}(x)=-4 / 5 \varepsilon x$ [2,20].

Passive tracer trajectories are obtained by integrating $\dot{\mathbf{x}}(t)=\mathbf{u}(\mathbf{x}(t), t)$ with the velocity at particle positions obtained by linear interpolation from the nearest grid points. The reported results are obtained averaging over a total number of about $3 \times 10^{5}$ particle pairs starting from initial separation $R(0)=L / 256$ and over 7 large scale eddy turnover times.

In Figure 2 we plot the second moment of relative dispersion $R^{2}(t)$. The Richardson $t^{3}$ law (4) is clearly observable although systematic deviations are detectable, in particular in the compensated plot. These deviations, observed also in kinematic simulations [10] and in twodimensional turbulence [8], are due to finite size effects. Consider a series of pair dispersion experiments, in each of which a couple of particles is released at time $t=0$ at initial separation $R(0)$. At a fixed time $t$ one performs an average over all realizations and computes $R^{2}(t)$. For $t$ small $R^{2}(t)$ is dominated by the initial distance, so that the $R^{2}(t)$-curve flattens. For large times some pairs might have reached a separation larger than the integral scale and thus show normal (not Richardson) diffusion, so that the $R^{2}(t)$-dependence flattens again. Under these conditions, a precise determination of the exponents and coefficients in (价), in particular the Richardson constant $C_{2}$, is very difficult.

The distribution of relative separations is plotted in Figure 3 for three different times. The form of the pdf is very close to the Richardson prediction (3) and excludes other distributions. Our result is the first direct numerical evidence of the substantial validity of Richardson's equation and gives support to recent experimental findings 沺. A closer inspection of Figure 3 reveals however that the self-similar evolution predicted by (Ii) is not exact. Again, the deviations from the distribution (3) are mostly due to finite Reynolds effects: because of the large tails, a large fraction of particles exits the inertial range after a very short time.

To overcome these difficulties in Lagrangian statistics, an alternative approach based on doubling time (or exit time) statistics has been recently proposed [10,21]. Given a set of thresholds $R_{n}=\rho^{n} R(0)$ within the inertial range, one computes the "doubling time" $T_{\rho}\left(R_{n}\right)$ defined as the time it takes for the particle pair separation to grow from threshold $R_{n}$ to the next one $R_{n+1}$. Averages are then performed over many dispersion experiments, i.e., particle pairs. The outstanding advantage of averaging at fixed scale separation, as opposite to a fixed time, is that it removes crossover effects since all sampled particle pairs belong to the same scales. In the simulations presented here, the value $\rho=1.2$ is used.

Let us first show how doubling time analysis can be used for estimating the Richardson constant $C_{2}$. Neglecting intermittency, the mean doubling time can be obtained from the first-passage problem for the Richardson diffusion equation (11) as [8]

$$
\left\langle T_{\rho}(R)\right\rangle=\frac{\rho^{2 / 3}-1}{2 k_{0} \varepsilon^{1 / 3} \rho^{2 / 3}} R^{2 / 3} .
$$

From (3) and (4) one has $C_{2}=\frac{1144}{81} k_{0}^{3}$. Comparison with (5) gives

$$
C_{2}=\frac{143}{81} \frac{\left(\rho^{2 / 3}-1\right)^{3}}{\rho^{2}} \frac{R^{2}}{\varepsilon\left\langle T_{\rho}\right\rangle^{3}}
$$

In the inset of Figure 4 we plot expression (6) which gives directly the value of $C_{2}$. Although the compensation is not perfect, it is possible to estimate the Richardson constant with much better accuracy than from the direct analysis of Figure 2. The resulting value, $C_{2}=0.55 \pm 0.1$, 
is remarkably close to the recent experimental finding [4]. The non perfect compensation is the consequence of intermittency.

Let us now discuss the issue of intermittency in more detail and concentrate on the behavior of the moments of inverse doubling times, $\left\langle\left(1 / T_{\rho}(R)\right)^{p}\right\rangle$. We expect for doubling time statistics a power-law behavior

$$
\left\langle\left(\frac{1}{T_{\rho}(R)}\right)^{p}\right\rangle \sim R^{\beta_{p}}
$$

with exponents $\beta_{p}$ connected to the exponents $\alpha_{n}$ in (4). Negative moments of doubling time are dominated by pairs which separate fast; this corresponds to positive moments of relative separation. Kolmogorov scaling, based on the dimensional analysis, gives $\left\langle\left(1 / T_{\rho}(R)\right)^{p}\right\rangle \sim$ $\varepsilon^{p / 3} R^{-2 p / 3}$ so that $\beta_{p}=-2 p / 3$. Intermittency can be taken into account by using the simple dimensional estimate for the doubling time, $T(R) \sim R / \delta u(R)$ which gives

$$
\beta_{p}=\zeta_{p}-p
$$

where $\zeta_{p}$ are the scaling exponents of the longitudinal structure functions. As a consequence of the Kolmogorov "4/5" law, $\zeta_{3}=1$ 20] and the doubling time exponent not affected by intermittency is $\beta_{3}=-2$ (again, the quantity not affected by intermittency depends on the first power of $\varepsilon$ ) 22.

In Figure 1 we plot the first moments of inverse doubling time (7) compensated with the Kolmogorov scaling $R^{-2 p / 3}$. The quality of the scaling is remarkable, especially if compared with the standard statistics of Figure 2. This allows to detect small deviations from dimensional scaling. Indeed, a closer inspection of Figure 4 reveals that the compensation is not perfect, the deviation being more evident for higher moments; this indicates the existence of Lagrangian intermittency.

Figure 5 shows some moments of the inverse doubling time, now compensated with best fit exponents $\beta_{p}$. The improvement with respect to Figure 4 demonstrates that the exponents in (7) are corrected in comparison to dimensional prediction. From the doubling time exponent $\beta_{p}$ we can obtain the Eulerian exponents $\zeta_{p}$ by inverting (8). The result shown in the inset of Figure 4 gives a set of exponents $\zeta_{p}$ which are remarkably close to "standard" structure function exponents in fully developed turbulence. We stress that, at the present resolution, the scaling of the Eulerian structure function is rather poor, thus a precise determination of $\zeta_{p}$ is possible only using indirect analysis, such as the ESS technique 23.

Let us summarize our findings. We have performed direct numerical simulations of a three-dimensional turbulent flow and concentrated on the problem of particles' dispersion. The overall dispersion behavior is welldescribed by the Richardson's equation, although some deviations (mostly caused by the finite-Reynolds nature of the simulations) are evident. The use of fixed-scale statistics (doubling-time distribution) instead of fixedtime ones removes to a large extent these restrictions, and gives a possibility to evaluation the Richardson's constant very accurately. Its value is $C_{2} \simeq 0.55$, in a close agreement with recent experimental findings. The discussion of the inverse moments of the doubling-time distributions unveils the role of Lagrangian intermittency in the two-particle dispersion. The values of the Lagrangian scaling exponents are connected with the Eulerian structure function exponents $\zeta_{p}$. The values of $\zeta_{p}$ obtained from the separation statistics are remarkably close to standard ones, known for fully developed turbulence. In the next future it will be probably possible to have experimental Lagrangian trajectories in high Reynolds number flows 24. It would be extremely interesting to check our findings in real fluid turbulence.

\section{ACKNOWLEDGMENTS}

We thank A. Celani and M. Cencini for useful comments and discussions. We acknowledge the allocation of computer resources from INFM Progetto Calcolo Parallelo.

[1] L.F. Richardson, Proc. Roy. Soc. A 110, 709 (1926).

[2] A. Monin and A. Yaglom, Statistical Fluid Mechanics (MIT Press, Cambridge, MA, 1975), Vol. 2.

[3] M.C. Jullien, J. Paret and P. Tabeling, Phys. Rev. Lett. 82, 2872 (1999).

[4] S. Ott and J. Mann J. Fluid Mech. 422, 207 (2000).

[5] F.W. Elliott, Jr. and A.J. Majda, Phys. Fluids 8, 1052 (1996).

[6] J.C.H. Fung and J.C. Vassilicos, Phys. Rev. E 57, 1677 (1998).

[7] N. Zovari and A. Babiano, Physica D 76, 318 (1994).

[8] G. Boffetta and I.M. Sokolov, Phys. Fluids (submitted, 2001), preprint nlin.CD/0009047.

[9] A.M. Obukhov, Izv. Akad. Nauk. SSSR 5, 453 (1941).

[10] G. Boffetta, A. Celani, A. Crisanti and A. Vulpiani, Phys. Rev. E 60, 6734 (1999).

[11] G.K. Batchelor, Proc. Camb. Phil. Soc. 48, 345 (1952).

[12] R. Kraichnan, Phys. Fluids 10, 1937 (1966).

[13] R. Kraichnan, Phys. Fluids 11, 945 (1968).

[14] G. Falkovich, K. Gawedzki and M. Vergassola, condmat/0105199 (2001).

[15] I.M. Sokolov, Phys. Rev. E 60, 5528 (1999).

[16] I.M. Sokolov, J. Klafter and A. Blumen, Phys. Rev. E 61, 2717 (2000).

[17] P. Castiglione, A. Mazzino, P. Muratore-Ginanneschi and A. Vulpiani, Physica D 134, 75 (1999).

[18] Shiyi Chen, Gary D. Doolen, Robert H. Kraichnan and Zhen-Su She, Phys. Fluids A 5, 458 (1993). 
[19] G. Falkovich, Phys. Fluids 6, 1411 (1994).

[20] U. Frisch, Turbulence, (Cambridge Univ. Press, Cambridge, 1995).

[21] V. Artale, G. Boffetta, A. Celani, M. Cencini and A. Vulpiani, Phys. Fluids A 9, 3162 (1997).

[22] It may seem that the -3 rd moment of $T_{\rho}(R)$, not affected by intermittency, may give a better estimate for $C_{2}$ than its first moment, Eq.(5). This is not the case, due to a remarkably strong $\rho$-dependence of $\left\langle\left(1 / T_{\rho}(R)\right)^{3}\right\rangle$ and to its strong sensitivity to temporal correlations. The corresponding results will be discussed elsewhere.

[23] R. Benzi, S. Ciliberto, R. Tripiccione, C. Baudet, F. Massaioli and S. Succi, Phys. Rev. E 48, R29 (1993).

[24] A. La Porta, G.A. Voth, A.M. Crawford, J. Alexander and E. Bodenschatz, Nature 409, 1017 (2001).

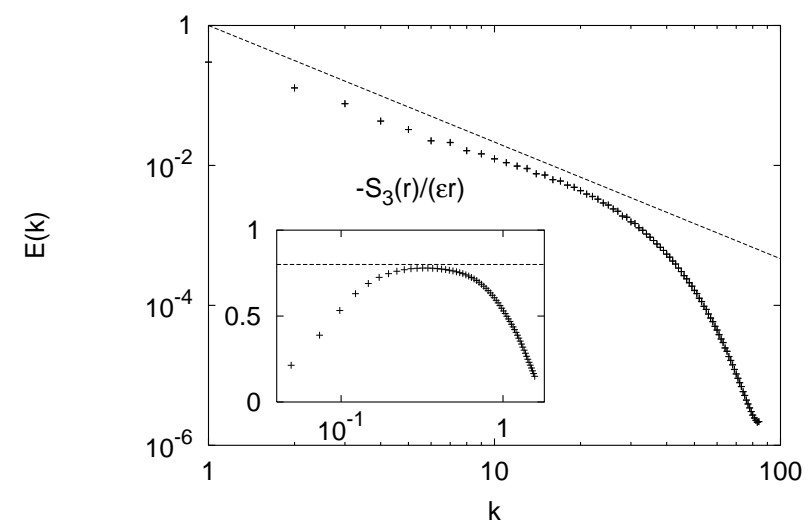

FIG. 1. Average energy spectrum $E(k)$. The dashed line has the Kolmogorov slope $-5 / 3$. In the inset it is shown the compensated third order longitudinal structure function. The dashed line represents the " $4 / 5$ " law.

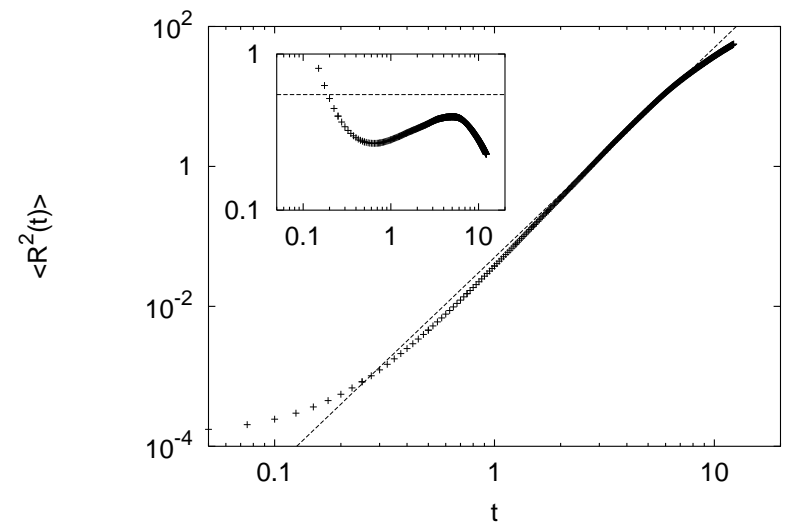

FIG. 2. Relative dispersion $R^{2}(t)$ versus time $t$. The dashed line is the Richardson $t^{3}$ law. In the inset we show the compensated plot $R^{2}(t) /\left(\varepsilon t^{3}\right)$ which should give the Richardson constant $C_{2}$. Because of the strong oscillation, a precise estimation of $C_{2}$ is difficult.

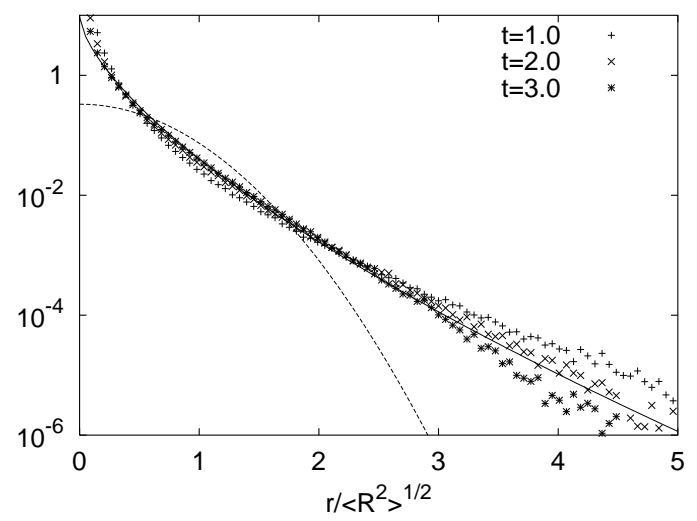

FIG. 3. Probability distribution function of relative separations at three different times. The continuous line is the Richardson prediction (3), the dashed line is the Gaussian distribution proposed by Batchelor.

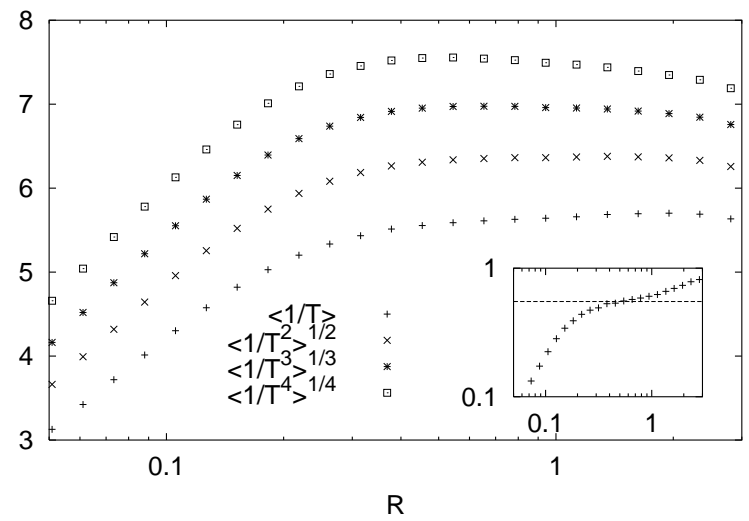

FIG. 4. First moments of the inverse doubling time $\left\langle(1 / T(R))^{p}\right\rangle$ compensated with Kolmogorov scaling $R^{-2 p / 3}$. Deviations from dimensional compensation are evident, in particular for $p=4$. In the inset we plot the compensated mean doubling time according to (6) together with the estimate corresponding to $C_{2} \simeq 0.55$. 


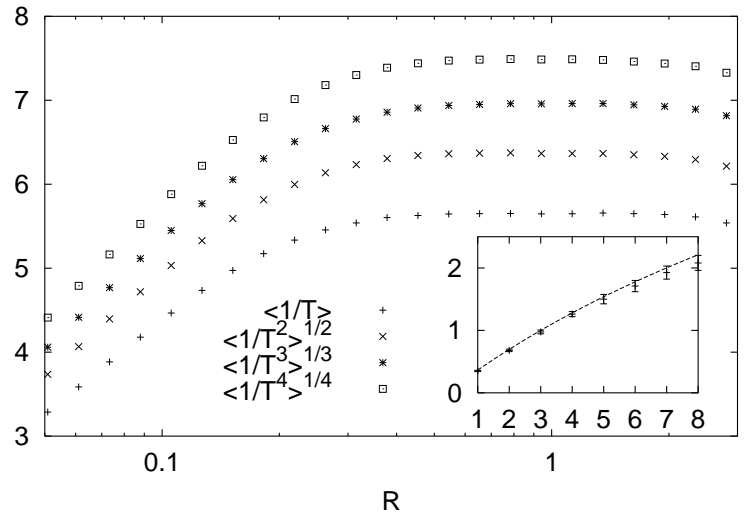

FIG. 5. First moments of the inverse doubling time $\left\langle(1 / T(R))^{p}\right\rangle$ compensated with best fit exponent $\beta_{p}$. Observe the improvement in the compensation with respect to Figure 4. In the inset we plot the structure function exponents estimated from $\zeta_{p}=p+\beta_{p}$. The line is the She-Leveque parameterization. 